\title{
Development of a learning progression for the formation of the Solar System
}

\author{
Julia Plummer ${ }^{1}$ \\ Alice Flarend \\ Chris Palma \\ KeriAnn Rubin \\ Brandon Botzer \\ Pennsylvania State University \\ ${ }^{1}$ Contact: jdp17@psu.edu
}

\section{Citation:}

Plummer, J.D., Flarend, A., Palma, C., Rubin, K., \& Botzer, B. (2013). Development of a learning progression for the formation of the Solar System. Presented at the annual meeting of the National Association for Research in Science Teaching, Rio Grande, PR. 


\begin{abstract}
This study describes the process of defining a hypothetical learning progression for astronomy around the big idea of Solar System formation. At the most sophisticated level of the learning progression, students can explain how the formation process leads to the current Solar System by considering how the planets formed from the collapse of a rotating cloud of gas and dust. In this first step towards understanding student progress in this domain, we interviewed middle school, high school, and college students $(\mathrm{N}=44)$, asking them to describe properties of the current Solar System and to explain how the Solar System was formed. Our analysis reveals potential levels of sophistication within the hypothetical learning progression while also revealing common alternative conceptions or areas of limited understanding that could form barriers to progress; many students' understanding of Solar System phenomena was limited by either alternative ideas about gravity or limited application of momentum in their explanations. Few students approached a scientific-level explanation, but their responses revealed possible stepping-stones that could be built upon with appropriate instruction. Our findings also point to critical deficiencies in how state and national standards address Solar System astronomy.

Keywords: astronomy, learning progression, measurement, middle and high school
\end{abstract}




\section{Introduction}

A significant body of research has shown that both children and adults have difficulty explaining astronomical phenomena, even those topics that are identified for elementary and middle school (Lelliott \& Rollnick, 2010). Policy documents, such as the Framework for K-12 Science Education (Framework; NRC, 2012), recommend that students become proficient in science in ways that go beyond basic facts and descriptions of phenomena; but how to successfully support students in developing an integrated view of astronomy remains an open question. Some may question a focus on astronomy given limited time in school for science. However, there are many reasons to engage students in learning astronomy. Longitudinal studies have found that high school students' spatial abilities, such as mental rotation and spatial visualization, predicts their future careers in science (Wai, Lubinski, \& Benbow, 2009). Spatial ability is malleable; thus, we can improve students' spatial abilities and thus potentially improve their chances to succeed in science (Uttal, Meadow, Tipton, Hand, Alden, et al., 2012). Learning astronomy can engage students in multiple dimensions of spatial thinking, such as issues related to size and scale and use of spatial reasoning by constructing explanations that account for different frames of reference (Mathewson, 1999; NRC, 2006; Plummer, 2013). Thus, instruction in astronomy - especially reform-based teaching that engages students in the type of mental manipulations that may improve spatial ability - could be an avenue towards improving their success in other areas of science. Astronomy instruction in middle and high school is also an opportunity for students to begin to apply other important science concepts, such as light, energy, and gravity, thus strengthening and deepening their understanding of those big ideas. Finally, many students are genuinely interested in astronomy and questions this field can answer, such as where did we come from and are we alone in the Universe? Thus, engaging students in astronomy instruction can help support their innate curiosity and provide them with a connection to a key area of science.

However, astronomy receives limited inclusion in classroom science instruction (Plummer \& Zahm, 2010), and, as with other areas of science, astronomy is often taught as fragmented, disconnected sets of facts that do not engage students in developing an integrated framework for explaining their own observations or exploring further (Corcoran et al., 2009; Kesidou \& Roseman, 2002; Schmidt, McKnight, \& Raizen, 1997). Learning progression research has the potential to help change the way we address astronomy in K-12 education, by guiding the development of research-based standards and curricula that support students' development of conceptual understanding of big ideas of science (Corcoran, Mosher, \& Rogat, 2009). Though the research community is still discussing how to design, revise, and validate learning progressions in science, there are several areas of common agreement. Learning progressions describe how students may grow in sophistication towards a big idea in science (e.g. Corcoran et al., 2009; NRC, 2007). These big ideas describe unifying concepts that help make sense of a broad variety of phenomena, offering robust explanatory power for the world around us (Smith et al., 2006). A learning progression describes how intermediate levels of sophistication can be valued and built upon by instruction across many grades, rather than only focusing on alternative ideas and scientific ideas. Finally, learning progressions are viewed as hypothetical models of learning describing "the typical levels that students' understanding might be expected to go through given instructional exposure" appropriate to the phenomena and experiences needed to make progress in their explanations (Rogat et al., 2011, p. 4). There may be multiple paths that students will take from their novice ideas towards the upper levels of 
sophistication; however, this number is likely to be a relatively small set, as pathways are defined by the logic of the discipline, student cognition, and instructional design (Krajcik, 2011).

For our work, we chose to organize a learning progression around the formation of the Solar System because this theory unifies the disparate phenomena often taught in middle and high school astronomy. It was chosen as the "big idea" for this learning progression research because 1) it is important to the field of astronomy, 2) connections can be made to children's initial observations of the world, and 3) it can "explain multiple, unified astronomical phenomena such that learning to explain an individual phenomenon helps the learner build in sophistication toward the big idea and, thus, toward explanations of other phenomena" (Plummer, 2012, p. 79). In brief, the Solar System began forming when a large cloud of gas and dust collapsed due to the force of gravity. The cloud already had an initial angular momentum, and as it collapsed, it became denser but also began to rotate faster. The pressure at the center became great enough to form a star, our Sun; material that collapsed into a disk around the Sun collided, stuck together, and grew into planets. The formation model explains observations of the current Solar System: the differences in planet composition, the relative flatness of the Solar System, and the motions of the planets and other objects. Thus, by focusing on the formation model, we can help teachers and students pay attention to features of the Solar System that are connected and can be explained by the same unified theory while moving away from rote memorization of "planet facts." Learning to explain the Solar System formation also provides a context in which students can expand their application of fundamental theories of physics (gravity, velocity, acceleration, and angular momentum).

To build a complex learning progression around scientific principles and phenomena, we have adopted a framework of building a learning progression by examining progress in multiple dimensions (C. Anderson, personal communication) that combine to form an explanation for the formation of the Solar System. This process involves building learning progressions from multiple construct maps (Wilson, 2005); a construct map is similar to a learning progression in that it possesses an upper anchor (the goal-level understanding set by the discipline and societal expectations), a lower anchor (where students begin as they enter school), and descriptions of increasing sophistication in between. Wilson (2009) proposed building learning progressions through the development of construct maps that could then be aligned based on empirical evidence of how students make connections across content. Several previous studies have described the development of learning progressions around multiple constructions (e.g. Jin \& Anderson, 2012; Plummer, 2012; Plummer \& Krajcik, 2010; Shea \& Duncan, 2013; Songer, Kelcey, Gotwals, 2009). Shea and Duncan (2013) describe a process of developing learning progressions through the analysis of contingencies between construct maps within the larger learning progressions. Contingencies are the interrelationships or dependencies between constructs. One of the challenges researchers face in the development process is to determine where progress along one construct map may depend on progress along another construct map (Plummer \& Krajcik, 2010; Shea \& Duncan, 2013; Wilson, 2009). The directionality of these relationships may not be obvious when considering only an analysis of the discipline. While some constructs may develop in parallel, in other cases, a student may require a certain level of sophistication in one construct to gain traction in another construct (Shea \& Duncan, 2013).

Figure 1 shows the construct maps in the Solar System learning progression for which data has been gathered in the present study: gravity, physical properties (e.g., the composition of planets and the sun), dynamic properties (e.g., how and why planets orbit), and the process of the Solar System's formation. Understanding of other constructs is also necessary for progress up 
the learning progression - angular momentum, size \& scale, and time - but full construct maps for these have not yet been developed. While any one of these dimensions can be analyzed individually, development towards the understanding the formation of the Solar System requires the student to make connections between the dimensions.

The connections shown between the construct maps in Figure 1 show hypothetical relationships between the individual construct maps. The complex relationships we are beginning to uncover through our analysis of student ideas suggests potential ways that these constructs may be related. However, this work is still preliminary as our evidence is drawn only from a cross-age study; this work is in preparation for the next phase of the research in which we will study how specific instructional interventions move students through the conceptual space described in our hypothetical Solar System learning progression. One of the limitations of our current study is that few students are at the upper levels of the construct maps where these connections lie. Further, once we have students who have gone through the same instructional conditions, we can look for areas of growth in the students' knowledge - as measured by the construct maps - as well as areas where some students did not progress. This will help us identify connections that students make between the construct maps. We have revised our interview protocol based on what we are learning in this round of data analysis to better capture thinking at those potential connection points between construct maps.

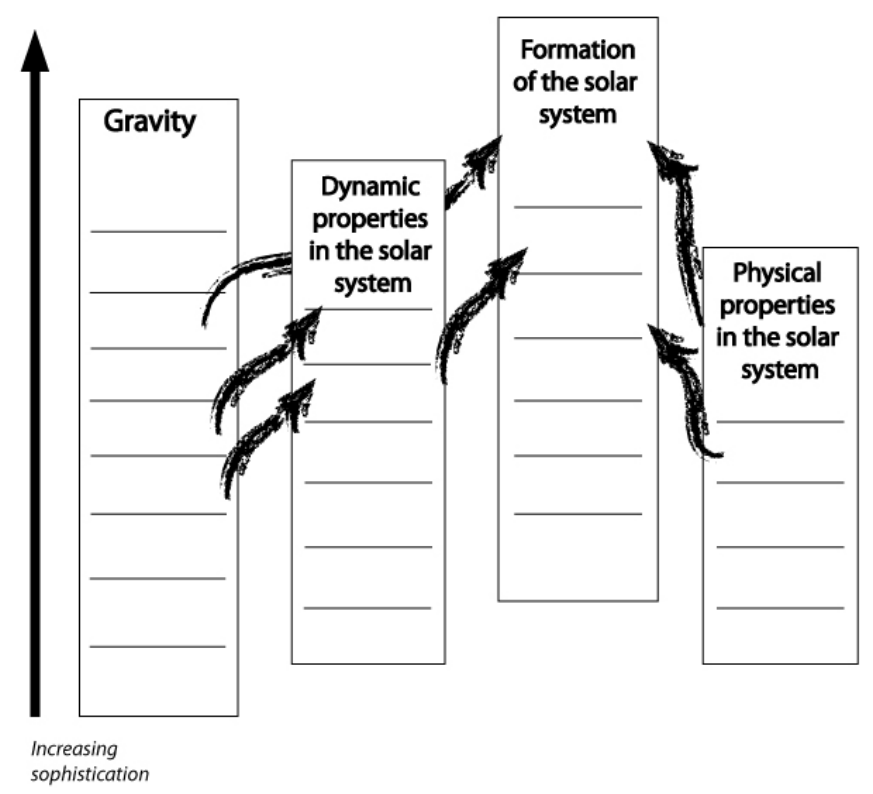

Figure 1. Four construct maps are shown: gravity, dynamic properties of the Solar System, formation of the Solar System, and physical properties in the Solar System. This representation shows a hypothetical depiction of how levels of sophistication increases upwards for each construct map and suggests that connections may be found between the construct maps.

\section{Methodology}

Students from middle school (6th grade), high school (9th - 12th grade), and college (introductory level) from urban, suburban, and rural locations in Pennsylvania were interviewed $(\mathrm{N}=44)$. The open-ended interview protocol engaged students in describing the current structure 
and motion of objects in the Solar System, formation of the Solar System, and gravity. Students were specifically asked questions that would allow them to describe any connections they could make between the way the Solar System is now, its formation, and the role of gravity.

Analysis began by identifying codes based on the research team's observations of a representative sample across grade levels. This was then applied to additional interviews, with new codes added and old codes refined as needed to capture students' thinking. Once this system of categories and codes had been applied to the interviews, we looked for ways to sort students' ideas along each construct with a goal of highlighting those aspects of students' thinking that moves them in productive ways towards the scientific idea. This led to the initial description of four construct maps: physical properties in the Solar System, dynamic properties in the Solar System, the role of gravity in the Solar System, and the formation of the Solar System. For each construct map, we aligned categories and codes associated with those big ideas into a succession of increasing sophistication. We also proposed very tentative connections - or places to look for connections - between the construct maps.

However, while the codes that make up these construct maps are based on our analysis of the student data, this is not sufficient to say that they are empirically based descriptions of possible levels in the construct maps. This is because of how the codes were grouped to form the levels. For example, in our first iteration of levels for the dynamic properties of the solar system construct map, we used codes from the categories for: their description of how the moon and planets move; their explanation for why the moon and planets move the way they do (e.g. why the orbit); the direction of the Moon and planets' orbits; and the overall shape of the solar system. Our initial description of how the codes could be used to describe students' developing sophistication for how and why the Moon and planets move fit the actual students well. But students with higher levels of understanding of those aspects of dynamical properties did not necessarily know that the planets all orbit the Sun in the same direction and on a plane. So, our original grouping of codes at specific levels was not supported by how the codes were grouped in individual students.

Therefore, our next step in developing the construct maps was to begin a process of applying our tentative construct maps to individual students to see how well they represented student thinking and to look for patterns across the students. The initial construct map level criteria were used as rubrics. The criteria (specific categories and corresponding codes) for each level were compared to individual student responses. This began an iterative cycle of comparing and revising the construct maps until the descriptions at each level of the construct map, based on our codes, were useful at representing the variations in student mental models observed in our interviews.

\section{Findings}

In the following sections, we examine characteristic examples of the patterns emerging from our analysis of students' explanations to illustrate potential levels in the construct maps and the connections between those construct maps that help support the development of our hypothetical learning progression. At this time, we have sufficient evidence to suggest potential levels in two of the construct maps: dynamic properties and formation of the Solar System. Analysis is ongoing for the remaining construct maps (gravity and physical properties); additional information can be shared upon request. 


\section{Dynamical Properties of Objects in the Solar System}

Students' understanding of how and why objects move in the Solar System was assessed through a series of questions that began with asking them to draw a picture of the Solar System. They were then prompted to describe how they think the Moon and planets may move and then to explain why the objects move the way that they do. At the upper anchor of this construct map, students can describe the Moon orbiting the Earth and the planets orbiting the Sun. Gravity holds the Moon in a constant near-circular orbit about the Earth and its inertia keeps it from crashing into the Earth. A similar explanation is applied to the planets' orbits about the Sun.

Table 1. Construct map for the dynamic properties in the Solar System. These levels describe students' ideas $(\mathrm{N}=43)$ about how and why the Moon and planets move in the Solar System.

\begin{tabular}{|c|c|c|c|c|}
\hline Level & Description for Level A & Description for Level B & $\begin{array}{c}\text { Level } \\
\text { A }\end{array}$ & $\begin{array}{c}\text { Level } \\
\text { B }\end{array}$ \\
\hline 4 & $\begin{array}{l}\text { 4A: Orbits in the Solar System } \\
\text { are the result of a balance } \\
\text { between the object's tangential } \\
\text { velocity and the gravitational } \\
\text { force between the object and } \\
\text { body it is orbiting (applied to } \\
\text { planets and/or Moon). Planets } \\
\text { and Moon all orbit in same } \\
\text { direction; Solar System is flat. }\end{array}$ & $\begin{array}{l}\text { 4B: Orbits in the Solar System are } \\
\text { the result of a balance between the } \\
\text { object's tangential velocity and the } \\
\text { gravitational force between the } \\
\text { object and body it is orbiting } \\
\text { (applied to planets and/or } \\
\text { Moon). Unclear or non-normative } \\
\text { reasoning for the shape of the Solar } \\
\text { System and/or the direction of } \\
\text { planetary orbits. }\end{array}$ & 0 & 3 \\
\hline 3 & $\begin{array}{l}\text { 3A: Orbits in the Solar System } \\
\text { are close to a flat plane, in the } \\
\text { same direction, and the result of } \\
\text { the gravitational force between } \\
\text { objects, holding one in orbit } \\
\text { about another. Unclear or non- } \\
\text { normative reasoning for why } \\
\text { objects do not crash into object } \\
\text { they orbit. Planets and Moon all } \\
\text { orbit in same direction; Solar } \\
\text { System is flat. }\end{array}$ & $\begin{array}{l}\text { 3B: Orbits in the Solar System are } \\
\text { result of the gravitational force } \\
\text { between objects, holding one in } \\
\text { orbit about another. Unclear or } \\
\text { non-normative reasoning for why } \\
\text { objects do not crash into object they } \\
\text { orbit, for the shape of the Solar } \\
\text { System, and for the direction of } \\
\text { planetary orbits. }\end{array}$ & 8 & 5 \\
\hline 2 & $\begin{array}{l}\text { 2A: Planets orbit about the Sun } \\
\text { in distinct orbits, in the same } \\
\text { direction. Student provides non- } \\
\text { normative reasoning for why } \\
\text { objects maintain their orbits. } \\
\text { Planets and Moon all orbit in } \\
\text { same direction; Solar System is } \\
\text { flat. }\end{array}$ & $\begin{array}{l}\text { 2B: The planets orbit the Sun and } \\
\text { the Moon orbits the Earth, but } \\
\text { student provides non-normative } \\
\text { ideas about the orbital direction, } \\
\text { shape of the Solar System, and } \\
\text { reasoning for why objects maintain } \\
\text { their orbits. }\end{array}$ & 0 & 8 \\
\hline 1 & \multicolumn{2}{|c|}{$\begin{array}{l}\text { The Moon does not orbit the Earth and/or the planets do not move or } \\
\text { do not move along distinct orbits about the Sun. }\end{array}$} & \multicolumn{2}{|c|}{19} \\
\hline
\end{tabular}


At Level 1, students do not describe the Moon as orbiting the Earth or do not provide distinct orbits for the planets around the Sun. With few exceptions, these students also cannot use scientific descriptions of how gravity works to account for how objects move in the Solar System (Table 1). Beyond Level 1, we have split the construct map into two paths. Both paths trace the progress in describing how the Moon and planets orbit and their use of gravity and momentum in their explanations. The split occurs because of how they described the direction of orbits and the shape of the Solar System. Our analysis revealed that students are capable of having sophisticated descriptions of planets orbiting due to the Sun's gravitational pull, but not understanding that the planets all orbit in the same direction and exist on a single, relatively thin, plane.

Moving up the construct map to Level 2, students describe planets orbiting the Sun in distinct orbits. While an important increase in sophistication, this is still a descriptive level of understanding. At this level, students continue to use non-normative explanations for why the Moon and the planets orbit. For example, one student discussed the existence of a magnetic-like force holding the Moon in orbit around the Earth. Thus, the first two levels show progress from non-normative to normative descriptions of orbital motion. Once this is established, the upper two levels describe increased sophistication in the explanation for the orbits. Students found the use of gravitational attraction to explain why the planets maintain their orbits to be more challenging than simply describing their orbits (not accounting for differences in direction).

At Level 3, students use the gravitational attraction between the Earth and Moon and between the planets and Sun to explain their orbits; but they cannot provide a normative explanation for why planets do not crash into the Sun. For example, Dana (11 ${ }^{\text {th }}$ grade; ID \#26) was asked why the moon does not crash into the Earth: "Because, well from the gravitation and the Earth has, they're very far from each other so I don't think they could crash into each other." Nathan $\left(12^{\text {th }}\right.$ grade; ID \#30) believes there is a resistive force between the Moon and Earth: "[The Moon] has its own gravity force, that, it doesn't suck in - it also fights [hits one fist into the other palm]."

At Level 4, students provide a normative view of why planets maintain stable orbits around the Sun that includes both inertia and gravity, such as the response from Michael $\left(11^{\text {th }}\right.$ grade, $\# 42)$ :

Sun is the most massive thing in our Solar System so it pulls all the other planets around it. I guess the planets were in motion so the reason they go in an ellipse is they're trying to continue their motion but then gravity pulls them back again. I guess, an object in motion stays in motion. It keeps trying to keep its path but the gravity continues to pull it back. -- Michael

This level of understanding was not typical of the students we interviewed and reveals an important barrier to progress. Many students, who knew gravity has a role in the planets' orbits, could not explain that planets orbit due to the combined effect of gravity and inertia (Level 3). The missing piece was inertia. This difficulty in integrating the balance between initial velocity and gravitational force to explain orbits led us to examine how the standards are written. The Pennsylvania State Science Standards focus on gravity with no indication of the role of the planets' initial velocity and the balance between gravity and inertia: "Recognize the role of gravity as a force that pulls all things on or near the Earth toward the center of the Earth and in the formation of the Solar System and the motion of objects in the Solar System." Similarly, the Framework does not address the role of this balance or how the orbits initially formed. 
Some students were able to reach the upper levels of the construct map without also understanding the direction of planets' orbits and the flatness of the Solar System; thus, we split the construct map from Levels 2-4. In our next phases of analysis, we will consider how those variables are tied to the students' explanations for how the Solar System formed. It may be that progress along the "A" strand of this construct map is necessary to build towards integrating the current Solar System with the formation model in students' explanations.

\section{Formation of the Solar System}

Students' understanding of the formation of the Solar System was assessed based on a series of questions that first asked them to discuss how they believed the Solar System to have formed and then to interpret a series of four illustrations depicting the scientific model of how the Solar System was formed (Figure 2). Students were asked to interpret the illustration and suggest what they think might be happening and how this relates to their understanding of the Solar System's formation. Students' initial explanations and later analysis of the images were highly consistent.

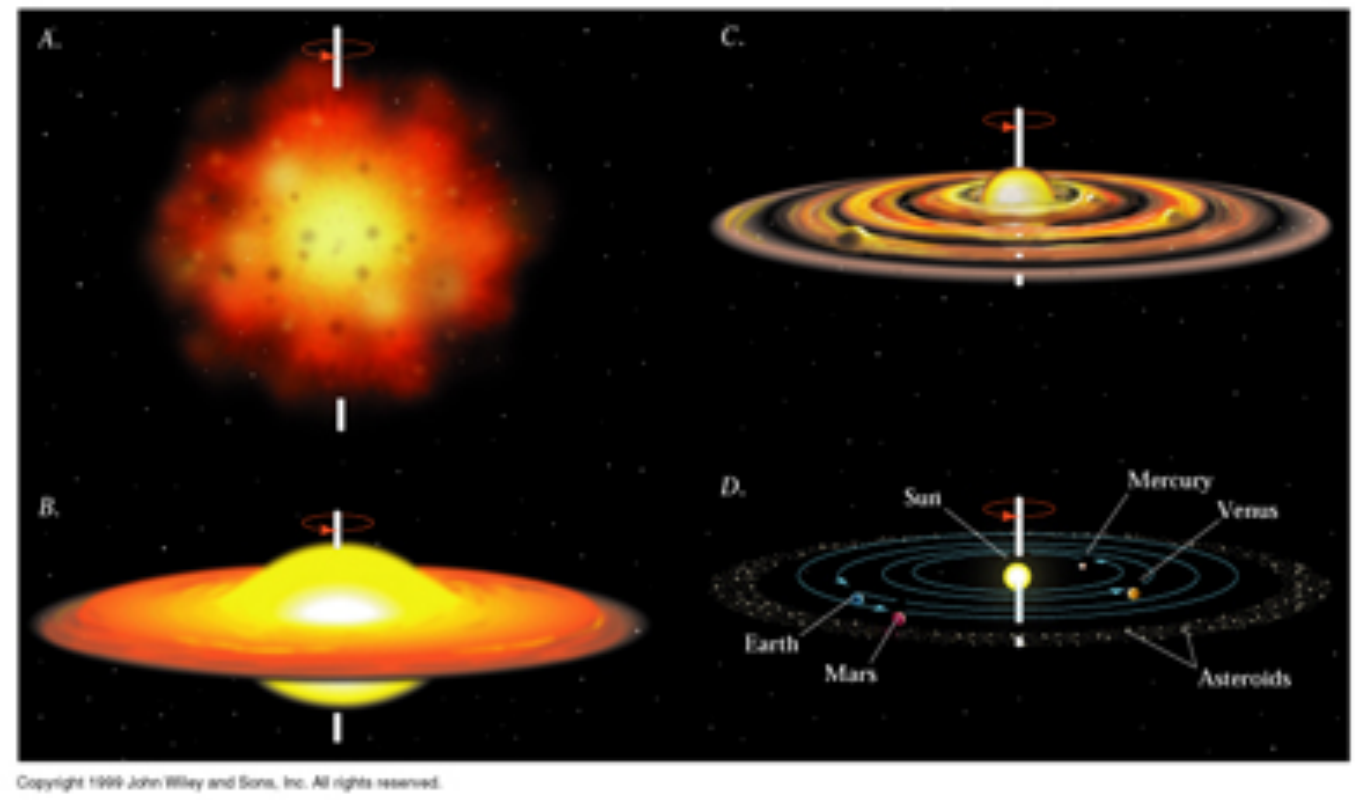

Figure 2. These four images were shown to the students. However, we cut the images apart and rescaled them such that the initial image of the gas cloud was larger than the subsequent disk images, to illustrate the collapse of a large cloud into the Solar System.

At the upper anchor of this construct map, students can explain how the formation process leads to the current Solar System by applying an understanding of how gravity and initial motion can be used to explain the process by which a cloud of gas and dust collapsed into a flat system of planets orbiting central star. Further, this explanation should account for how accretion resulted in a gradual building of the Sun or planets as material "stuck" together in the collapsing cloud. Table 2 illustrates the major aspects of increasing sophistication of students' explanations for the Formation of the Solar System. 
Table 2. Features of students' explanations for the formation of the Solar System

\begin{tabular}{|c|l|c|}
\hline Level & Description & $\begin{array}{c}\text { Students } \\
(\mathrm{N}=25)\end{array}$ \\
\hline 6 & $\begin{array}{l}\text { The Solar System formed from the accretion of microscopic materials such } \\
\text { as gas, rock and/or dust. Gravity caused the collapse of this material into } \\
\text { the Sun and planets. The Sun and planets formed at the same time. }\end{array}$ & 3 \\
\hline 5 & $\begin{array}{l}\text { The Solar System formed from macroscopic materials such as gas, rocks, } \\
\text { meteors and fragments. Formation occurred after an explosion and then } \\
\text { contraction. There was accretion of this macroscopic material into the } \\
\text { planets. Gravity caused the collapse. Sun and planets may have formed } \\
\text { separately. }\end{array}$ & 5 \\
\hline 4 & $\begin{array}{l}\text { The Solar System formed from materials such as gas, rock and/or dust. } \\
\text { Formation occurred due to a vague accretion-like process. There may have } \\
\text { been an explosion that started the process. Gravity holds system together } \\
\text { during formation, but does not cause accretion. }\end{array}$ & 3 \\
\hline 3 & $\begin{array}{l}\text { At least part of the Solar System formed from materials such as gas, rock } \\
\text { and/or dust. But no mechanism for where material came from or how it } \\
\text { comes together. Gravity plays a role in the formation or maintenance of } \\
\text { the whole system. }\end{array}$ & 4 \\
\hline 2 & $\begin{array}{l}\text { Planets, or material to make the planets, were drawn in by Sun. Gravity } \\
\text { may play a role by holding system together, during formation. }\end{array}$ & 3 \\
\hline 1 & The Solar System began as an explosion. Gravity played no role. & 7 \\
\hline
\end{tabular}

\section{Gravity's role in the Solar System's formation}

At the least sophisticated level, Level 1, lie students who believe that the Solar System began as an explosion and that gravity plays no role in this process. For example, Ryan (11 grade, ID\#40) a physics student at Level 1 explains the formation as "a big explosion.... molten rocks slowly cooled down and formed into giant [spheres]." A common theme observed in students at this level was conflating the formation of the Universe (Big Bang) with the formation of the Solar System; many middle school, high school, and college students believe that the Big Bang describes the formation of the Solar System (Prather, Slater, \& Offerdahl, 2002). During the interview, students were given a series of four images showing cartoon representations of the scientific model of the formation of the Solar System (Figure 2). The series of images start with a large cloud of gas and dust, followed by an image of a smaller cloud that has begun to collapse with a bulge at the center, followed by a still smaller cloud in which the disk is clearly visible and pockets of gas are starting to collect where the planets will form, and finally a version of the current Solar System showing the Sun and planets in orbits. The image shows the initial cloud being smaller than the final Solar System; this is an incorrect representation. Therefore, the version of this image that we showed to the students during the interview was manipulated to show the initial cloud to be largest in the first image, getting smaller in the subsequent images as the cloud collapses and the planets and Sun form. Despite the fact that the progression of images shows a system that gets smaller and smaller, students often still interpreted this as an explosion. Students often will interpret their observations in ways that are consistent with their prior conceptual beliefs (Eberbach \& Crowley, 2009). 
One of the ways that we have to measure progress is through students' use of gravity in explaining the Solar System's formation. At Level 1, students do not use gravity as part of their explanation but in Level 2 and 3 they consider gravity playing a role in the large-scale process of formation or maintenance, such as holding the system together. Similarly, at Level 4 gravity holds the system together during formation but does not cause the collapse and accretion of material. Cullen ( $11^{\text {th }}$ grade, ID\#38), a physics student at Level 4, knows that the Solar System formed from gas and dust, knows that gravity works at a distance, and is directly proportional to the objects' mass. However, Cullen was unable to explain gravity's role in the formation process because he believes that gases do not have mass and therefore do not gravitationally attract.

Moving up to Level 5 and 6, gravity is involved in some stage of the collapse of the initial material into the Sun and planets. For example, Nina $\left(6^{\text {th }}\right.$ grade, ID\#2) is at Level 5; she first describes how planets formed from the build up of lava, which was attracted by the gravitational force. She describes gravity as "pushing down" to flatten the Solar System into a disk. When asked why the Solar System is flat, she says: "the gravity in the space like pushed down on every part here, and smooshed it." Allyson (college, ID\#17) at Level 6 initially does not discuss gravity in how she explained the formation of the Solar System. But when she is asked if gravity plays a role, she uses gravity to explain why the Solar System is flat:

Interviewer: Do you see the role of gravity [in the pictures of the Solar System formation]?

Allyson: Yeah, right here, when you're getting spun like that, the gravity pushes everything to the outside.

Interviewer: Gravity pushes stuff that way?

Allyson: It's holding it in right there. It's pulling it up. Gravity is here, but like its um keeping everything in a tight circle like that, and it's also pulling everything together so it's like a flatter pancake.

However, she does not use gravity to account for the reason why the cloud collapsed in the first place.

Interviewer: Was this thing [points to the first image of the gas cloud] rotating?

Allyson: That collapsed on itself. So something pushes it when it gets cold so it collapses in on itself then when it collapses in on itself it begins its spiral rotation.

Interviewer: Why does it collapse?

Allyson: Because cold gas can't support itself.

Interviewer: So it's cooling down?

Allyson: Mmm-hmm. It collapses in on itself. Well, it's still really hot. I'm saying cold

gas, I mean like colder, not freezing. It can't support itself and so then it starts collapsing and rotates.

She remembers the normative portions of the scientific model: gas clouds cannot collapse to form new Solar Systems unless they are cold and that this may have been induced by something "bumping" into the molecular cloud (such as a supernova or neighboring molecular cloud). But she does not invoke the balance between the cloud's gas pressure with the opposing force of gravity acting to cause the collapse over dense regions.

\section{Accretion processes in the formation of the Solar System}

Students' progress was also measured by looking at how they explained the accretion of materials into the current objects in the Solar System. At the lowest level, students were unable 
to account for how the initial materials formed planets; some described just an initial explosion. Others described the planets and Sun as forming separately and then coming together into the system. More sophisticated explanations describe a process of growth or accretion. Matthew ( $6^{\text {th }}$ grade, ID\#20), a middle school student at Level 3, recognized some components of accretion but was unable to explain further details: "By gas and dust just coming together, well that's how the Sun was formed, just gas and dust just formed it and came together like that [gestures by bringing hands together in front of him]. It was just made."

At the upper anchor, students use the formation model to make connections between the initial conditions and materials of the solar nebula and the current physical properties of the Solar System. This level of sophistication was not observed in the students from our sample. Few students made significant progress in using the formation to account for current properties and locations of the planets. Cullen (11 ${ }^{\text {th }}$ grade, ID\#38), Level 4, attempted to make sense of this began by describing the formation process as "condensing from a cloud of gas"; he suggested that the reason why the initial gas cloud became solid planets was because the gas condensed, or changed states, by cooling off.

However these explanations do not account for how larger objects grew from the initial condition of gas and dust. Level 5 and 6 are distinguished by whether students saw the formation of planets as starting with macroscopic objects or microscopic particles. For example, Nina described above - described planets as accreting from hot lava. Jared (high school, \#48) was placed in Level 5 because he believes the Solar System began with an explosion: "At one point it just exploded and sucked back in and just like formed." However, unlike other Level 5 students, he used a microscopic accretion model.

Interviewer: What has to happen to create a Sun or a planet?

Jared: Different gases, the Sun filled with gas, like if matter and space [puts his hands together as if making something] formed together.

Therefore, Jared is a student at the boundary between levels 5 and 6; he cannot move up until he shifts his explanation away from an explosion to more normative descriptions of the initial conditions of the Solar System's formation.

A few students, like Allyson (college, ID\#17), provided a more sophisticated explanation attempt to account for building up of smaller particles, while also distinguishing between the Big Bang and Solar System formation:

Once the Big Bang created all those clouds, there was a huge cloud... once it becomes colder gas... it starts spiraling, or collapsing in on each other and it can't stop. Once its collapsing it's gaining rotating and getting hotter. All the dust and particles start forming together. The hot particles fuse together. That's how all the sun and planets form at the same time. -- Allyson

This explanation reveals some alternative conceptions as she focuses on the materials heating up as a precursor for accretion processes, rather than considering gravitational or electrostatic forces. However, she does use gravity to explain why the solar system flattened into a disk which helps to put her into the highest level in the construct map.

\section{Discussion}

Our findings contribute to understanding how students develop increasingly sophisticated explanations for Solar System phenomena. While other studies have explored pieces of this learning progression, such as students' ideas about the nature of the Solar System (Sharp \& 
Kuerbis, 2005) or gravity's role in astronomy (Williamson \& Willoughby, 2012), our work approaches from the perspective of integrating the many dimensions of Solar System astronomy to help students develop explanations of why the properties of the Solar System exist the way that they do currently. This approach is consistent with the Framework, which states: "The history of the universe, and of the structures and objects within it, can be deciphered using observations of their present condition together with knowledge of physics and chemistry" (2012, p. 173). We ultimately hope to draw attention to the importance of designing curriculum and standards for secondary school astronomy in ways that will produce a more integrated understanding of phenomena. This integrated systems approach to teaching and learning about the formation of the Solar System is significantly different from how many teachers, students, and standards talk about Solar System astronomy. For example, the PA State Science Standards are a set of individual statements that do not clarify the relationship between Solar System formation and the current motions and compositions of planets; the new Framework does little to make this connection explicit, beyond that both are governed by gravity.

However, by learning to explain the formation of the Solar System, students begin to address some of the Next Generation Science Standards (NGSS). For example, the initial Framework for developing these new standards describes the following unpacking of conceptual understanding of forces: "Newton's law of universal gravitation provides the mathematical model to describe and predict the effects of gravitational forces between distant objects. These long-range gravitational interactions govern the evolution and maintenance of large-scale structures in the universe (e.g., the Solar System, galaxies) and the patterns of motion within them" (NRC, 2012, p. 116-117). Progress within the dynamic properties construct map moves students towards the NGSS middle school standard for forces and interactions: "Construct and present oral or written arguments that use evidence to support the claim that gravitational interactions determine the motion of systems of objects in space" (2013, p. 39).

The NGSS high school standards go on to describe expectations for students understanding of the formation of the Solar System: "Construct explanations from data for the formation of the Solar System based on space exploration and astronomical evidence of the composition, structure, and motions of Solar System bodies" (P. 94). Clarifications to the standard suggest that potential evidence for how the Solar System formed may include that the Sun and planets rotate in the same direction as the planets orbit the Sun, the composition of meteorites which reveal the initial composition of the Solar System, and the distribution of planet compositions, with rocky and metal-rich planets close to the sun and increasing inclusion of ice moving farther out in the Solar System. Our current Solar System formation construct map may help us understand where students need to be in order to take this next step of constructing an explanation that uses the model of formation.

Considering the NGSS standard for Solar System formation also helps us consider ways to analyze how the construct maps come together as well as future steps for the research. First, the high school level standard for Solar System formation specifically suggests assessing students' ability to use the formation model to explain the current dynamic and physical properties of the Solar System. Therefore, one of the links we need to look for in students' thinking is how and when they can construct evidence-based explanations for the Solar System's formation. For example, one of the elements of the dynamic properties construct map is whether or not students believe that the planets all orbit in the same direction. An important connection between this construct map and the formation construct map is using the collapse of a spinning cloud to account for why the planets would be expected to all be orbiting in the same direction. 
Second, the NGSS can help us shape our next steps in this research study. Now that we have a sense of the types of practices that have been crossed with these core science concepts for the Standards, we can use what we have learned about students' conceptual development to now assess how students' engagement with the science practices progresses along with their understanding of the content.

Our findings also have implications for how Solar System astronomy is taught. First, helping students make connections across the dimensions could begin by organizing instruction on the properties of planets around the features explained by the formation model instead of having individual students learning about planets as isolated objects. Teachers should focus on helping students see ways of grouping planets that lead them to questions about how the Solar System formed. Second, more effort should be made to teach planetary motion using a force and motion framework that connects to how they are learning these concepts in earth-based contexts; our interviews suggest that students have primarily been learning about orbits in terms of gravity, rather than a richer application of physics that helps them understand the role of momentum. Flarend and Palma (in review) recommend that students have the opportunity to engage in discourse around data on planets' orbital velocities and distance in order to construct scientific explanations based on a claims-evidence-reasoning format (McNeill \& Krajcik, 2012). Students can use their data to construct an argument for how a planet's velocity depends on distance and justify this using the scientific model for gravity falling off with distance. In this way, students can integrate descriptive knowledge of planetary motion with scientific theory and move up the Solar System learning progression.

\section{Conclusion}

This work is an attempt to illustrate the importance of looking at concepts that students are expected to learn in the middle grades from the perspective of the current scientific model of Solar System formation. Uncovering how progress in students' understanding of fundamental principles of physics and spatial thinking (time and scale) relates to their progress in explaining phenomena of astronomy adds to the field's understanding of how to make connections across the science curriculum. Our results reveal that many students across middle school, high school, and college find constructing explanations for Solar System phenomena (e.g. orbits and formation) to be challenging. Their knowledge is often descriptive and lacks a foundation in key aspects of physics. Similarly, their descriptive knowledge is often incomplete or not focused towards aspects of the Solar System model that would be productive towards further progress in connecting to how the Solar System formed.

\section{Acknowledgements}

This work was funded by an NSF Targeted Math Science Partnership award (DUE\#0962792).

\section{References}

Corcoran, T., Mosher, F. A., \& Rogat, A. (2009, May). Learning progressions in science: An evidence-based approach to reform (CPRE Research Report \#RR-63). Philadelphia, PA: Consortium for Policy Research in Education. 
Eberbach, C. \& Crowley, K. (2009). From everyday to scientific observation: How children learn to observe the biologist's world. Review of Educational Research, 73, 39-68.

Flarend, A. \& Palma, C. (in review). The role of gravity in planetary orbits. The Earth Scientist.

Jin, H. \& Anderson, C.W. (2012). A learning progression for energy in socio-ecological systems. Journal of Research in Science Teaching, 49, 1149-1180.

Kesidou, S., \& Roseman, J. E. (2002). How well do middle school science programs measure up? Findings from Project 2061's curriculum review. Journal of Research in Science Teaching, 39, 522-549.

Krajcik, J.S. (2011). Learning progressions provide road maps for the development and validity of assessments and curriculum materials, Measurement, 9, 155-158.

Lelliott, A. \& Rollnick, M. (2010). Big ideas: A review of astronomy education research 19742008. International Journal of Science Education, 32, p. 1771-1799.

Mathewson, J. H. (1999). Visual-Spatial Thinking: An Aspect of Science Overlooked by Educators. Science Education, 83, 33-54.

McNeill, K. L. and Krajcik J. S. 2012 Supporting Grade $5-8$ Students in Constructing Explanations in Science Boston, MA: Pearson.

National Research Council (2012). A framework for K-12 science education: Practices, crosscutting concepts, and core ideas. Washington, DC: National Academies Press.

National Research Council. (2007). Taking science to school: Learning and teaching science in grades K-8. Washington, DC: National Academies Press.

National Research Council (2006). Learning To Think Spatially, Washington, DC: National Academies Press.

National Research Council. (1996). National science education standards. Washington, DC: National Academy Press.

Plummer, J.D. (2013). Spatial reasoning as the dimension of progress in an astronomy learning progression. To be presented at the American Education Research Association, San Francisco, CA.

Plummer, J.D. (2012). Challenges in developing and validating an astronomy learning progression. In A. Alonzo and A.W. Gotwals (Eds.), Learning Progressions in Science: Current Challenges and Future Directions, pp. 77-100, Sense Publishers: Rotterdam, The Netherlands.

Plummer, J.D. \& Krajcik, J.S. (2010). Building a Learning Progression for Celestial Motion: Elementary Levels from an Earth-Based Perspective. Journal of Research in Science Teaching, 47(7), 768-787.

Plummer, J. D., \& Zahm, V. (2010). Covering the standards: Astronomy teachers' preparation and beliefs. Astronomy Education Review, 9(1). doi:10.3847/AER2009077

Prather, E.E., Slater, T.F., \& Offerdahl, E.G. (2002). Hints of a fundamental misconception in cosmology. Astronomy Education Review, 1, 28-34.

Rogat, Anderson, Foster, Goldberg, Hicks, et al. (2011). Developing Learning Progressions in Support of the New Science Standards. Consortium for Policy Research in Education.

Sharp, J. \& Kuerbis, P. (2005). Children's ideas about the Solar System and the chaos in learning science. Science Education, 90, 124-147.

Shea, N.A. \& Duncan, R.G. (2013). From theory to data: The process of refining learning progressions. Journal of the Learning Sciences, 22, 7-32.

Smith, C. L., Wiser, M., Anderson, C. W., \& Krajcik, J. (2006). Implications of research on children's learning for standards and assessment: A proposed learning progression for matter and the atomic-molecular theory. Measurement: Interdisciplinary Research and 
Perspectives, 4, 1-98.

Songer, N. B., Kelcey, B., \& Gotwals, A. W. (2009). How and when does complex reasoning occur? Journal of Research in Science Teaching, 46(6), 610-631.

Uttal, D., Meadow, N., Tipton, E., Hand, L., Alden, A., Warren, C., \& Newcombe, N. (in press). The malleability of spatial skills: A meta-analysis of training studies, Psychological Bulletin.

Wai, J., Lubinski, \& Benbow, C.P. (2009). Spatial ability for STEM domains: Aligning over 50 years of cumulative psychological knowledge solidifies its importance. Journal of Educational Psychology, 101, 817-835.

Williamson, K.E., \& Willoughby, S. (2012). Student understanding of gravity in introductory college astronomy. Astronomy Education Review, 11, 10.3847/AER2011025

Wilson, M. (2005). Constructing measures: An item response modeling approach. Mahwah, NJ: Lawrence Erlbaum Associates.

Wilson, M. (2009). Measuring progressions: Assessment structures underlying a learning progression. Journal of Research in Science Teaching, 46, 716-730. 